\section{§3. Degradation of Optical Reflectivity of In-vessel Mirror Materials by Helium Bombardment}

Kajita, S., Saeki, T., Ohno, N. (Nagoya Univ.), Tokitani, M.

In future experimental fusion devices, many in-vessel mirrors will be used for optical diagnostics. It is generally recognized that metallic mirrors are used for them, and the candidate materials for the first mirror are molybdenum, tungsten, copper, stainless steel, and rhodium. Although it has been found that neutron irradiation does not have a significant influence on the optical reflectivity, there is concern that the reflectivity decreases by the exposure to charge-exchange neutrals, which cause erosion and deposition. In the present report, we will show the effect of low-energy helium bombardment to molybdenum and rhodium from the experiments conducted in a linear plasma device.

The experiments were performed in the linear divertor simulator NAGDIS (NAGoya DIvertor Simulator)-I. The specimens were exposed to the helium plasma in the NAGDIS-I. The surface temperature and incident ion energy were controlled by changing the helium flux to the specimen. The incident ion energy was controlled by electrically biasing the specimen, and was $\sim 50 \mathrm{eV}$. The wavelength dependence of optical reflectivity was measured with a spectrophotometer (Nihon Bunkosya: ARV-47S) for the wavelength from 200 to $900 \mathrm{~nm}$.

Figure 1(a) shows the wavelength dependences of the optical reflectivity of Mo-1 and Mo-2. The irradiation temperatures for Mo-1 and Mo-2 are 1200 and $1500 \mathrm{~K}$, respectively, and the helium ion fluences for Mo-1 and Mo-2 were $1.8 \times 10^{26} \mathrm{~m}^{-2}$ and $1.1 \times 10^{27} \mathrm{~m}^{-2}$, respectively. The reflectivity considerably decreased by the surface modifications due to the helium irradiation. The optical reflectivity was less than $10 \%$ for Mo-1, whereas, for Mo-2, it decreased to almost zero. Non-uniformity of Mo-1 surface is effectively averaged for the reflectivity measurement, because the spot size in the measurement is much larger than the crystal grain. Figure 1(b) shows the reduction factor of the reflectivity for Mo-1. Here, the reduction factor was defined as the original reflectivity divided by the decreased reflectivity. At $900 \mathrm{~nm}$, the reduction factor was approximately 5; it increased as decreasing the wavelength, and was 30 at $200 \mathrm{~nm}$. In general, optical reflectivity is strongly altered when the size of the structure on the surface is less than several times the wavelength [1]. From Fig. 1(b), the size of the structure is less than $1 \mu \mathrm{m}$; moreover, there may exist much finer structure on the surface.

Figure 2(a) shows the wavelength dependences of nonirradiated rhodium, helium irradiated rhodium (Rh-1), and helium irradiated molybdenum (Mo-3). The incident ion energy, surface temperature, and helium ion fluence for Rh-1 and Mo-3 were, respectively, $45 \mathrm{eV}, 600 \mathrm{~K}$, and $8 \times 10^{24} \mathrm{~m}^{-2}$ and $45 \mathrm{eV}, 850 \mathrm{~K}$, and $3.7 \times 10^{25} \mathrm{~m}^{-2}$. The exposure to the helium plasmas also decreased the optical reflectivity significantly for both rhodium and molybdenum even at those temperatures. Figure 2(b) shows the reduction factors for Rh-1 and Mo-3. In both cases, the factor increases as decreasing the wavelength. The factor increases considerably particularly in the range of less than $300 \mathrm{~nm}$.

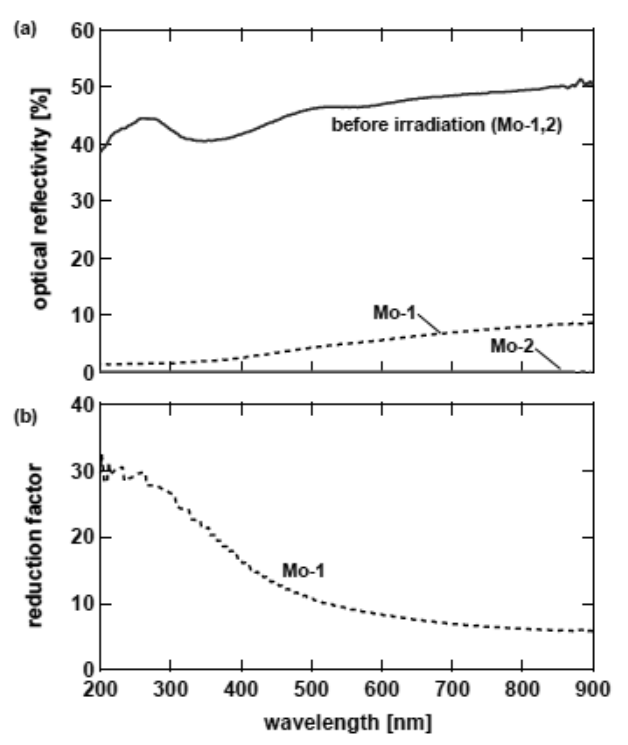

Fig. 1 (a)Wavelength dependences of the optical reflectivity of molybdenum samples before irradiation and after helium irradiations (Mo-1 and Mo-2) are shown. (b) Wavelength dependence of the reduction rate of optical reflectivity for Mo-1. Here, the reduction rate was defined as the reflectivity before irradiation divided by the reflectivity of Mo-1. The irradiation temperatures for Mo-1 and Mo-2 are 1200 and $1500 \mathrm{~K}$, respectively.

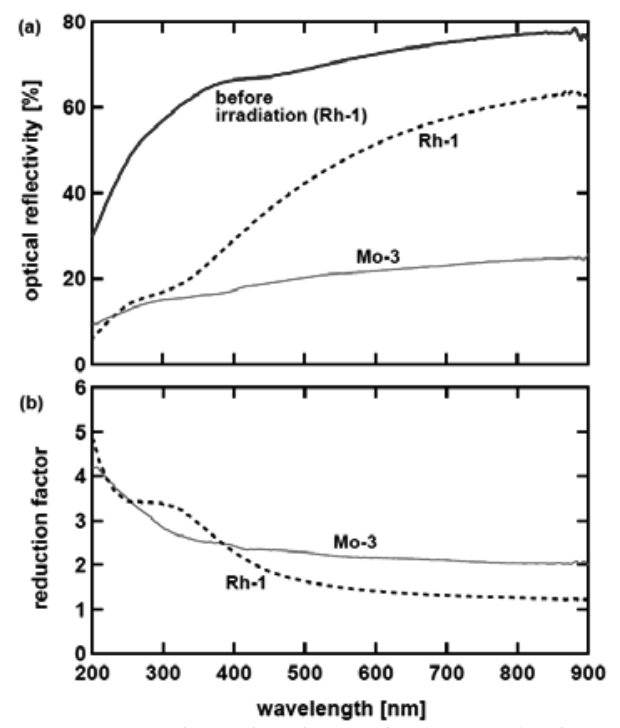

Fig. 2 (a) Wavelength dependences of the optical reflectivity of rhodium and molybdenum samples before irradiation and after helium irradiation (Rh-1 and Mo-3) are shown. (b) Wavelength dependence of the reduction rate of optical reflectivity for Rh-1 and Mo-3. The irradiation temperatures for Rh-1 and Mo-3 are 600 and $850 \mathrm{~K}$, respectively.

1) E. Rephaeli, S. Fan, Appl. Phys. Lett. 92 (2008) 211107. 